\title{
Characterization on Irregular Tight Wavelet Frames with Matrix Dilations ${ }^{\star}$
}

\author{
Deyun Yang ${ }^{1,2}$, Zhengliang Huan ${ }^{1}$, Zhanjie Song $^{3}$, and Hongxiang Yang ${ }^{1}$ \\ ${ }^{1}$ Department of Information Science and Technology, Taishan University, Taian \\ 271000, China \\ nkuydy@163.com \\ ${ }^{2}$ School of Control Science and Engineering, Shandong University, Jinan 250061, \\ China \\ ${ }^{3}$ School of Science, Tianjin University, Tianjin 300072, China
}

\begin{abstract}
There are many results in one dimensional wavelet frame theory in recent years. However, since there are some essential differences in high dimensional cases, the classical methods for one dimensional regular wavelet frames are unsuitable for the cases. In this paper, under some conditions on matrix-dilated sequences, a characterization formula for irregular tight frames of matrix-dilated wavelets is developed. It is based on the regular formulation by Chui, Czaja, Maggioni, Weiss, and on the recent multivariate results by Yang and Zhou.
\end{abstract}

Keywords: Irregular frame, Tight wavelet frame, Matrix dilations, Bessel sequences.

\section{Characterization on Irregular Tight Wavelet Frames with Matrix-Dilations}

Assume $\psi, \tilde{\psi} \in L^{2}\left(\mathbf{R}^{n}\right),\left\{A_{j}\right\}_{j \in \mathbf{Z}}$ is a real $n \times n$ matrix sequence, $B$ is a real $n \times n$ nonsingular matrix. $A^{-*}$ is the transpose of $A^{-1}$. Let

$$
\Psi=\left\{\psi_{j, k}: j \in \mathbf{Z}, k \in \mathbf{Z}^{n}\right\}, \tilde{\Psi}=\left\{\tilde{\psi}_{j, k}: j \in \mathbf{Z}, k \in \mathbf{Z}^{n}\right\},
$$

where $\psi_{j, k}(x)=\left|\operatorname{det} A_{j}\right|^{-\frac{1}{2}} \psi\left(A_{j}^{-*} x-B k\right)$. If $\left\{\psi_{j, k}: j \in \mathbf{Z}, k \in \mathbf{Z}^{n}\right\}$ is a tight frame for $L^{2}\left(\mathbf{R}^{n}\right)$, then $\psi$ is called a tight frame function with respect to dilation sequence $\left\{A_{j}\right\}_{j \in \mathbf{Z}}$. There are some results in high dimensional wavelet frame theory in recent years (see [1] - [7] ). Let $P(f, g)=\sum_{j \in \mathbf{Z}, k \in \mathbf{Z}^{n}}\left\langle f, \psi_{j, k}\right\rangle\left\langle\tilde{\psi}_{j, k}, g\right\rangle$, $f, g \in L^{2}\left(\mathbf{R}^{n}\right)$. Now we consider the following subset of $L^{2}\left(\mathbf{R}^{n}\right)$ :

$$
\mathcal{B}=\left\{f \in L^{2}\left(\mathbf{R}^{n}\right): \widehat{f} \in L^{\infty}\left(\mathbf{R}^{n}\right), \widehat{f} \text { has compact support. }\right\}
$$

The following lemma comes from [2].

\footnotetext{
* This work was supported by the National Natural Science Foundation of China (Grant No. 60572113).
} 
Lemma 1. Two families $\left\{e_{\alpha}: \alpha \in \mathcal{A}\right\}$ and $\left\{\tilde{e}_{\alpha}: \alpha \in \mathcal{A}\right\}$ constitute a dual pair if and only if they are Bessel sequences and satisfy

$$
P(f, g):=\sum_{\alpha \in \mathcal{A}}\left\langle f, e_{\alpha}\right\rangle\left\langle\tilde{e}_{\alpha}, g\right\rangle=\langle f, g\rangle,
$$

for all $f, g$ in a dense subset of $\mathcal{H}$.

Given $\left\{A_{j}\right\}_{j \in \mathbf{Z}}$ and $B$, we need the following notations:

$$
\begin{gathered}
\wedge=\left\{\alpha \in \mathbf{R}^{n}: \alpha=A_{j}^{-*} m,(j, m) \in \mathbf{Z} \times B^{-*}\left(\mathbf{Z}^{n}\right)\right\}, \\
I(\alpha)=\left\{(j, m) \in \mathbf{Z} \times B^{-*}\left(\mathbf{Z}^{n}\right): \alpha=A_{j}^{-*} m\right\} .
\end{gathered}
$$

Assume that $\left\{A_{j}\right\}_{j \in \mathbf{Z}}$ satisfy the following conditions: there exist $\beta \in(0,1)$, $q \in \mathbf{Z}_{+}$such that

$$
\begin{aligned}
& \left\|A_{j} A_{j+1}^{-1}\right\| \leq 1,\left\|A_{j} A_{j+q}^{-1}\right\| \leq \beta \text { for any } j \in \mathbf{Z}, \\
& \left\|A_{j+1}^{-1} A_{j}\right\| \leq 1,\left\|A_{j+q}^{-1} A_{j}\right\| \leq \beta \quad \text { for any } j \in \mathbf{Z} .
\end{aligned}
$$

Then we have

Theorem 2. Suppose that $\psi, \tilde{\psi} \in L^{2}\left(\mathbf{R}^{n}\right)$ have the property that the following two functions are locally integrable:

$$
\sum_{j \in \mathbf{Z}}\left|\widehat{\psi}\left(A_{j} \omega\right)\right|^{2}, \sum_{j \in \mathbf{Z}}\left|\widehat{\widetilde{\psi}}\left(A_{j} \omega\right)\right|^{2} .
$$

Then for $f, g \in \mathcal{B}, P(f, g)$ converges absolutely. Moreover,

$$
\frac{1}{|\operatorname{det} B|} \sum_{(j, m) \in I(\alpha)} \widehat{\tilde{\psi}}\left(A_{j} \omega\right) \overline{\widehat{\psi}\left(A_{j}\left(\omega+A_{j}^{-1} m\right)\right)}=\delta_{\alpha, 0},
$$

for a.e. $\omega \in \mathbf{R}^{n}$ and for all $\alpha \in \wedge$, if and only if $P(f, g)=\langle f, g\rangle$ for all $f, g \in \mathcal{B}$. By Theorem 2 and Lemma 1, the following two theorems are the immediate consequences.

Theorem 3. Assume that $\Psi, \tilde{\Psi} \subset L^{2}\left(\mathbf{R}^{n}\right)$ are two Bessel sequences,

$$
\sum_{j \in \mathbf{Z}}\left|\widehat{\psi}\left(A_{j} \omega\right)\right|^{2}, \sum_{j \in \mathbf{Z}}\left|\widehat{\widetilde{\psi}}\left(A_{j} \omega\right)\right|^{2}
$$

are locally integrable. Then $\Psi$ and $\widetilde{\Psi}$ constitute a pair of dual frames for $L^{2}\left(\mathbf{R}^{n}\right)$ if and only if

$$
\frac{1}{|\operatorname{det} B|} \sum_{(j, m) \in I(\alpha)} \widehat{\widetilde{\psi}}\left(A_{j} \omega\right) \overline{\widehat{\psi}\left(A_{j}\left(\omega+A_{j}^{-1} m\right)\right)}=\delta_{\alpha, 0},
$$

for a.e. $\omega \in \mathbf{R}^{n}$ and for all $\alpha \in \wedge$. 
Theorem 4. $\psi \in L^{2}\left(\mathbf{R}^{n}\right)$ satisfies

$$
\frac{1}{|\operatorname{det} B|} \sum_{(j, m) \in I(\alpha)} \widehat{\psi}\left(A_{j} \omega\right) \overline{\widehat{\psi}\left(A_{j}\left(\omega+A_{j}^{-1} m\right)\right)}=\delta_{\alpha, 0},
$$

for a.e. $\omega \in \mathbf{R}^{n}$ and for all $\alpha \in \wedge$, if and only if $\left\{\psi_{j, k}\right\}_{j, k}$ is a tight frame with constant 1 for $L^{2}\left(\mathbf{R}^{n}\right)$.

\section{Proof of Main Results}

In fact, we only give the proof of Theorem 2 ,

Proof of Theorem 2; We first prove that $P(f, g)$ is absolutely convergent. Now, let

$$
G_{j}:=\sum_{k \in \mathbf{Z}^{n}}\left\langle f, \psi_{j, k}\right\rangle\left\langle\tilde{\psi}_{j, k}, f\right\rangle, j \in \mathbf{Z} .
$$

Using the Parseval identity, it is easy to show

$$
G_{j}=\frac{1}{|\operatorname{det} B|} \int_{\mathbf{R}^{n}} \overline{\widehat{f}(\omega)} \widehat{\tilde{\psi}}\left(A_{j} \omega\right)\left(\sum_{s \in \mathbf{Z}^{n}} \widehat{f}\left(\omega+A_{j}^{-1} B^{-*} s\right) \overline{\widehat{\psi}\left(A_{j} \omega+B^{-*} s\right)}\right) d \omega .
$$

Thus, we would like to show that $\sum_{j \in \mathbf{Z}} G_{j}$ is absolutely convergent. To do so, it is enough to show that the following two series are absolutely convergent:

$$
I:=\sum_{j \in \mathbf{Z}} \int_{\mathbf{R}^{n}} \overline{\widehat{f}(\omega)} \widehat{\tilde{\psi}}\left(A_{j} \omega\right) \widehat{f}(\omega) \overline{\hat{\psi}\left(A_{j} \omega\right)} d \omega
$$

and

$$
I I:=\int_{\mathbf{R}^{n}} \overline{\widehat{f}(\omega)} \widehat{\tilde{\psi}}\left(A_{j} \omega\right)\left(\sum_{s \in \mathbf{Z}^{n} \backslash\{0\}} \widehat{f}\left(\omega+A_{j}^{-1} B^{-*} s\right) \overline{\widehat{\psi}\left(A_{j} \omega+B^{-*} s\right)}\right) d \omega .
$$

Since

$$
\left|\widehat{\tilde{\psi}}\left(A_{j} \omega\right) \widehat{\psi}\left(A_{j} \omega+B^{-*} s\right)\right| \leq \frac{1}{2}\left(\left|\widehat{\tilde{\psi}}\left(A_{j} \omega\right)\right|^{2}+\left|\widehat{\psi}\left(A_{j} \omega+B^{-*} s\right)\right|^{2}\right),
$$

It follows from (4) and the conditions on $\psi, \tilde{\psi}, f$ that $I$ is absolutely convergent.

On the other hand, for $h \in\{\psi, \tilde{\psi}\}$,

$$
\begin{aligned}
& \sum_{j \in \mathbf{Z}, s \in \mathbf{Z}^{n} \backslash\{0\}} \int_{\mathbf{R}^{n}}\left|\widehat{f}(\omega) \widehat{f}\left(\omega+A_{j}^{-1} B^{-*} s\right)\right|\left|\widehat{h}\left(A_{j} \omega\right)\right|^{2} d \omega \\
= & \int_{\mathbf{R}^{n}}\left(\sum_{j \in \mathbf{Z}, s \in \mathbf{Z}^{n} \backslash\{0\}} \frac{1}{\left|\operatorname{det} A_{j}\right|}\left|\widehat{f}\left(A_{j}^{-1} \omega\right) \widehat{f}\left(A_{j}^{-1}\left(\omega+B^{-*} s\right)\right)\right|\right)|\widehat{h}(\omega)|^{2} d \omega .(5)
\end{aligned}
$$

Since $f \in \mathcal{B}$, it follows from (2) and (3) that there exists constant $C_{0}>0$ such that for each $j \in \mathbf{Z}, \omega \in \mathbf{R}^{n}$, the number of $s \in \mathbf{Z}^{n} \backslash\{0\}$ satisfying 


$$
\widehat{f}\left(A_{j}^{-1} \omega\right) \widehat{f}\left(A_{j}^{-1} \omega+A_{j}^{-1} B^{-*} s\right) \neq 0
$$

is less than $C_{0}\left|\operatorname{det} A_{j}\right|$. Then

$$
\sum_{j \in \mathbf{Z}, s \in \mathbf{Z}^{n} \backslash\{0\}} \frac{1}{\left|\operatorname{det} A_{j}\right|}\left|\widehat{f}\left(A_{j}^{-1} \omega\right) \widehat{f}\left(A_{j}^{-1}\left(\omega+B^{-*} s\right)\right)\right| \leq C \sum_{j \in \mathbf{Z}} \chi_{F}\left(A_{j}^{-1} \omega\right),
$$

where $C=C_{0}\|\hat{f}\|_{\infty}^{2}$ and $F$ is compact in $\mathbf{R}^{n} \backslash\{0\}$. By (2) and (3), $\left\{A_{j}\right\}_{j \in \mathbf{Z}}$ is an MFS (see [4). Therefore there exists constant $K>0$ such that

$$
\sum_{j \in \mathbf{Z}} \chi_{F}\left(A_{j}^{-1} \omega\right) \leq K \text { for any } \omega \in \mathbf{R}^{n} \backslash\{0\} .
$$

Now, it follows from (5), (6) and (7) that the series $I I$ is convergent. Hence, we can rearrange the series for $P(f, f)$, to obtain

$$
P(f, f)=\sum_{\alpha \in \wedge} \int_{\mathbf{R}^{n}} \overline{\hat{f}(\omega)} \hat{f}(\omega+\alpha)\left(\frac{1}{|\operatorname{det} B|} \sum_{(j, m) \in I(\alpha)} \widehat{\tilde{\psi}}\left(A_{j} \omega\right) \overline{\hat{\psi}\left(A_{j}(\omega+\alpha)\right)}\right) d \omega .
$$

Then using the polarization identity for the form $P$, we get the sufficiency of the theorem.

Next, we prove the necessary condition. From the above discussion, we have, let

$$
P(f, g)=M(f, g)+R(f, g),
$$

where

$$
M(f, g):=\frac{1}{|\operatorname{det} B|} \int_{\mathbf{R}^{n}} \overline{\widehat{g}(\omega)} \widehat{f}(\omega)\left(\sum_{j \in \mathbf{Z}} \widehat{\tilde{\psi}}\left(A_{j} \omega\right) \overline{\widehat{\psi}\left(A_{j} \omega\right)}\right) d \omega
$$

and

$$
R(f, g):=\frac{1}{|\operatorname{det} B|} \sum_{\alpha \in \wedge \backslash\{0\}} \int_{\mathbf{R}^{n}} \overline{\widehat{g}(\omega)} \widehat{f}(\omega+\alpha) \sum_{(j, m) \in I(\alpha)} \widehat{\tilde{\psi}}\left(A_{j} \omega\right) \overline{\hat{\psi}\left(A_{j}(\omega+\alpha)\right)} d \omega .
$$

Now, fix $\omega_{0} \in \mathbf{R}^{n} \backslash\{0\}$ and let

$$
\widehat{f}_{1}(\omega)=\frac{1}{\mu\left(H_{k}\right)^{1 / 2}} \chi_{\omega_{0}+H_{k}}(\omega),
$$

where $\mu(\cdot)$ is the Lebesgue measure on $\mathbf{R}^{n}$ and

$$
H_{k}=A_{k}^{-1} \triangle, \triangle=\left\{\xi \in \mathbf{R}^{n}:|\xi|=1\right\} .
$$

Then

$$
M\left(f_{1}, f_{1}\right)=\frac{1}{|\operatorname{det} B| \mu\left(H_{k}\right)} \int_{\omega_{0}+H_{k}} \sum_{j \in \mathbf{Z}} \widehat{\tilde{\psi}}\left(A_{j} \omega\right) \overline{\widehat{\psi}\left(A_{j} \omega\right)} d \omega
$$


and

$$
\begin{aligned}
& \quad R\left(f_{1}, f_{1}\right) \mid \\
& \quad \leq \frac{1}{|\operatorname{det} B| \mu\left(H_{k}\right)} \sum_{\alpha \in \wedge \backslash\{0\}(j, m) \in I(\alpha)} \sum_{\left(\omega_{0}+H_{k}\right) \cap\left(\alpha+\omega_{0}+H_{k}\right)}\left|\widehat{\tilde{\psi}}\left(A_{j} \omega\right) \widehat{\psi}\left(A_{j}(\omega+\alpha)\right)\right| d \omega \\
& \quad \leq \frac{1}{|\operatorname{det} B| \mu\left(H_{k}\right)}\left(\sum_{\alpha \in \wedge \backslash\{0\}} \sum_{(j, m) \in I(\alpha)} \int_{\left(\omega_{0}+H_{k}\right) \cap\left(\alpha+\omega_{0}+H_{k}\right)}\left|\widehat{\tilde{\psi}}\left(A_{j} \omega\right)\right|^{2} d \omega\right)^{1 / 2} \\
& \quad \times\left(\sum_{\alpha \in \wedge \backslash\{0\}}\left|\widehat{\psi}\left(A_{j}(\omega+\alpha)\right)\right|^{2} d \omega\right)^{1 / 2} . \\
& \text { If }\left(\omega_{0}+H_{k}\right) \cap\left(\omega_{0}+\alpha+H_{k}\right) \neq \emptyset, \text { then } \alpha \in A_{k}^{-1}(\triangle-\triangle) \text { Thus for }(j, m) \in I(\alpha), \\
& m \in\left(A_{j} A_{k}^{-1}\left(\triangle-\triangle H_{k}\right) \cap\left(\alpha+\omega_{0}+H_{k}\right)\right.
\end{aligned}
$$

Using (2) and (3), there exists a constant $c>0$ such that $j \geq k+c$. However,

$$
\begin{aligned}
& \left|R\left(f_{1}, f_{1}\right)\right| \\
& \quad \leq \quad \frac{1}{|\operatorname{det} B| \mu\left(H_{k}\right)}\left(\sum_{j \geq k+c} \sum_{m \in Q_{j, k} \backslash\{0\}} \int_{\omega_{0}+H_{k}}\left|\widehat{\widetilde{\psi}}\left(A_{j} \omega\right)\right|^{2} d \omega\right)^{1 / 2} \\
& \left.\quad \times \quad \sum_{j \geq k+c}\left|\widehat{\psi}\left(A_{j}(\omega)\right)\right|^{2} d \omega\right)^{1 / 2} .
\end{aligned}
$$

For the first factor,

$$
\begin{aligned}
& \frac{1}{\mu\left(H_{k}\right)} \sum_{j \geq k+c} \sum_{m \in Q_{j, k} \backslash\{0\}} \int_{\omega_{0}+H_{k}}\left|\widehat{\tilde{\psi}}\left(A_{j} \omega\right)\right|^{2} d \omega \\
\leq & \left|\operatorname{det} A_{k}\right| \sum_{j \geq k+c} C\left|\operatorname{det}\left(A_{j} A_{k}^{-1}\right)\right|\left|\operatorname{det} A_{j}\right|^{-1} \int_{A_{j}\left(\omega_{0}+H_{k}\right)}|\widehat{\tilde{\psi}}(\omega)|^{2} d \omega \\
= & C \sum_{j \geq k+c} \int_{A_{j}\left(\omega_{0}+H_{k}\right)}|\widehat{\tilde{\psi}}(\omega)|^{2} d \omega,
\end{aligned}
$$

Here, we have used the fact $\#\left(Q_{j, k} \backslash\{0\}\right) \leq C \mu\left(A_{j} A_{k}^{-1}(\Delta-\Delta)\right)$, which can be obtained by (2), (3).

Similarly, we can estimate the second factor. Now, using (2) and (3), it is easy to prove that:

$\left(P_{1}\right)$ there exist $k_{1}, k_{2} \in \mathbf{Z}$ such that the intersection of any $k_{2}$ sets in $\left\{A_{j}\left(\omega_{0}+\right.\right.$ $\left.\left.H_{k}\right)\right\}_{k \geq k_{1}, j \geq k+c}$ is empty.

$\left(P_{2}\right)$ there exist constants $k_{3} \in \mathbf{Z}, \lambda>1$ such that for any $k \geq k_{3}, j \geq k+c$,

$$
A_{j}\left(\omega_{0}+H_{k}\right) \subset\left\{\omega:|\omega| \geq k_{3} \lambda^{j}\left|\omega_{0}\right|\right\}
$$


Using (8), (9), $\left(P_{1}\right)$ and $\left(P_{2}\right)$, we can get $\left|R\left(f_{1}, f_{1}\right)\right| \rightarrow 0$ when $k \rightarrow \infty$. Then, by Lebesgue theorem, we have

$$
\begin{aligned}
1 & =\lim _{k \rightarrow \infty} \frac{1}{|\operatorname{det} B| \mu\left(H_{k}\right)} \int_{\omega_{0}+H_{k}} \sum_{j \in \mathbf{Z}} \widehat{\tilde{\psi}}\left(A_{j} \omega\right) \overline{\widehat{\psi}\left(A_{j} \omega\right)} d \omega \\
& =\frac{1}{|\operatorname{det} B|} \sum_{j \in \mathbf{Z}} \widehat{\tilde{\psi}}\left(A_{j} \omega_{0}\right) \overline{\widehat{\psi}\left(A_{j} \omega_{0}\right)},
\end{aligned}
$$

which proves our claim for $\alpha=0$. This also shows that

$$
M(f, g)=\langle f, g\rangle
$$

for any $f, g \in \mathcal{B}$.

To complete the proof of our theorem, choose $\alpha_{0} \in \wedge \backslash\{0\}$, and write

$$
R(f, g)=R_{1}(f, g)+R_{2}(f, g)
$$

where

$$
\begin{gathered}
R_{1}(f, g)=\frac{1}{|\operatorname{det} B|} \int_{\mathbf{R}^{n}} \overline{\widehat{g}(\omega)} \widehat{f}\left(\omega+\alpha_{0}\right) \sum_{(j, m) \in I\left(\alpha_{0}\right)} \widehat{\widetilde{\psi}}\left(A_{j} \omega\right) \overline{\widehat{\psi}\left(A_{j}\left(\omega+\alpha_{0}\right)\right)} d \omega . \\
R_{2}(f, g)=\frac{1}{|\operatorname{det} B|} \sum_{\alpha \in \wedge \backslash\left\{0, \alpha_{0}\right\}} \int_{\mathbf{R}^{n}} \overline{\widehat{g}(\omega)} \widehat{f}(\omega+\alpha) \sum_{(j, m) \in I(\alpha)} \widehat{\tilde{\psi}}\left(A_{j} \omega\right) \overline{\widehat{\psi}\left(A_{j}(\omega+\alpha)\right)} d \omega .
\end{gathered}
$$

Next, let $\omega_{0} \in \mathbf{R}^{n} \backslash\{0\}$ be any Lebesgue point of functions $\sum_{j \in \mathbf{Z}}\left|\widehat{\psi}\left(A_{j} \omega\right)\right|^{2}$ and $\sum_{j \in \mathbf{Z}}\left|\widehat{\tilde{\psi}}\left(A_{j} \omega\right)\right|^{2}$. For fixed $k \in \mathbf{Z}$, we define $f_{2}, g_{2}$ as follows:

$$
\widehat{f_{2}}\left(\omega+\alpha_{0}\right)=\frac{1}{\mu\left(H_{k}\right)^{1 / 2}} \chi_{\omega_{0}+H_{k}}(\omega), \quad \widehat{g_{2}}(\omega)=\frac{1}{\mu\left(H_{k}\right)^{1 / 2}} \chi_{\omega_{0}+H_{k}}(\omega) .
$$

Then, using Lebesgue Theorem, we have

$$
\lim _{k \rightarrow \infty} R_{1}\left(f_{2}, g_{2}\right)=\frac{1}{|\operatorname{det} B|} \sum_{(j, m) \in I\left(\alpha_{0}\right)} \widehat{\tilde{\psi}}\left(A_{j} \omega_{0}\right) \overline{\widehat{\psi}\left(A_{j}\left(\omega_{0}+\alpha_{0}\right)\right)} .
$$

To estimate $R_{2}\left(f_{2}, g_{2}\right)$, we note that if $\widehat{g_{2}}(\omega) \widehat{f_{2}}(\omega+\alpha) \neq 0$, then

$$
\alpha \in \alpha_{0}+A_{k}^{-1}(\triangle-\triangle)
$$

Since $\alpha=A_{j}^{-1} m \in \wedge \backslash\left\{0, \alpha_{0}\right\}$, it follows from (2), (3) that there exist $J_{0} \in \mathbf{Z}$ such that for any $j \leq J_{0}, m \in B^{-*}\left(\mathbf{Z}^{n}\right) \backslash\{0\}, A_{j}^{-1} m \notin \alpha_{0}+D_{k}$, where

$$
D_{k}=A_{k}^{-1}(\triangle-\triangle)
$$


Hence $R_{2}\left(f_{2}, g_{2}\right)$ can be rearranged as

$$
\begin{aligned}
& R_{2}\left(f_{2}, g_{2}\right) \\
& =\frac{1}{|\operatorname{det} B|} \sum_{j \geq J_{1}} \sum_{m \in\left(A_{j} \alpha_{0}+A_{j} D_{k}\right) \backslash\{0\}} \int_{\mathbf{R}^{n}} \overline{\widehat{g_{2}}(\omega)} \widehat{f}_{2}(\omega+\alpha) \widehat{\tilde{\psi}}\left(A_{j} \omega\right) \overline{\widehat{\psi}\left(A_{j}(\omega+\alpha)\right)} d \omega \\
& +\frac{1}{|\operatorname{det} B|} \sum_{j=J_{0}}^{J_{1}} \sum_{m \in\left(A_{j} \alpha_{0}+A_{j} D_{k}\right) \backslash\{0\}} \int_{\mathbf{R}^{n}} \overline{\widehat{g_{2}}(\omega)} \widehat{f_{2}}(\omega+\alpha) \widehat{\tilde{\psi}}\left(A_{j} \omega\right) \overline{\widehat{\psi}\left(A_{j}(\omega+\alpha)\right)} d \omega \\
& =R_{2,1}\left(f_{2}, g_{2}\right)+R_{2,2}\left(f_{2}, g_{2}\right),
\end{aligned}
$$

where $J_{1} \in \mathbf{Z}$. Using (2) and (3), when $k$ is large enough, for each $j\left(J_{0} \leq j \leq J_{1}\right)$ the number of $m$ satisfying $m \in\left(A_{j} \alpha_{0}+A_{j} D_{k}\right)$ is a constant which is not related on $k$. Thus, by Lebesgue theorem, we have $\lim _{k \rightarrow \infty} R_{2,2}\left(f_{2}, g_{2}\right)=0$.

To estimate $R_{2,1}\left(f_{2}, g_{2}\right)$, we would like to prove that for each $\varepsilon>0$ and $k$ which is large enough, there exists $J_{1} \in \mathbf{Z}$ such that $R_{2,1}\left(f_{2}, g_{2}\right) \leq \varepsilon$.

In fact, similar to $R\left(f_{1}, f_{1}\right)$, we have

$$
\begin{aligned}
R_{2,1}\left(f_{2}, g_{2}\right) & \leq \frac{1}{|\operatorname{det} B|\left|H_{k}\right|}\left(\sum_{j \geq J_{1}} \sum_{m \in\left(A_{j} \alpha_{0}+A_{j} D_{k}\right) \backslash\{0\}} \int_{\omega_{0}+H_{k}}\left|\widehat{\tilde{\psi}}\left(A_{j} \omega\right)\right|^{2} d \omega\right)^{1 / 2} \\
& \times\left(\sum_{j \geq J_{1}} \sum_{m \in\left(A_{j} \alpha_{0}+A_{j} D_{k}\right) \backslash\{0\}}\left|\widehat{\psi}\left(A_{j} \omega\right)\right|^{2} d \omega\right)^{1 / 2} \cdot
\end{aligned}
$$

Therefore it is enough to estimate just one of these factors.

In fact, fix any $k$ which is large enough. Using the conditions (2) and (3), there exists a constant $C$ such that for any $j \geq J_{1}$,

$$
\#\left(\left(A_{j} \alpha_{0}+A_{j} D_{k}\right) \cap B^{-T}\left(\mathbf{Z}^{n}\right)\right) \leq 1+C\left|\operatorname{det} A_{j}\right|\left|\operatorname{det} A_{k}\right|^{-1},
$$

where $\#(\cdot)$ is the number of elements in a given set. Then

$$
\begin{aligned}
& \frac{1}{\left|H_{k}\right|} \sum_{j \geq J_{1}} \sum_{m \in\left(A_{j} \alpha_{0}+A_{j} D_{k}\right) \backslash\{0\}} \int_{\omega_{0}+H_{k}}\left|\widehat{\tilde{\psi}}\left(A_{j} \omega\right)\right|^{2} d \omega \\
\leq & \frac{1}{\left|H_{k}\right|} \sum_{j \geq J_{1}}\left(1+C\left|\operatorname{det} A_{j}\right|\left|\operatorname{det} A_{k}\right|^{-1}\right) \int_{\omega_{0}+H_{k}}\left|\widehat{\tilde{\psi}}\left(A_{j} \omega\right)\right|^{2} d \omega \\
= & \sum_{j \geq J_{1}} \frac{1}{\left|H_{k}\right|} \int_{\omega_{0}+H_{k}}\left|\widehat{\tilde{\psi}}\left(A_{j} \omega\right)\right|^{2} d \omega+\sum_{j \geq J_{1}} \int_{A_{j}\left(\omega_{0}+H_{k}\right)}|\widehat{\tilde{\psi}}(\omega)|^{2} d \omega .
\end{aligned}
$$

Let $J_{1} \in \mathbf{Z}$ such that $\sum_{j \geq J_{1}}\left|\widehat{\psi}\left(A_{j} \omega_{0}\right)\right|^{2}<\varepsilon$. Then

$$
\lim _{k \rightarrow \infty} \sum_{j \geq J_{1}} \frac{1}{\mu\left(H_{k}\right)} \int_{\omega_{0}+H_{k}}\left|\widehat{\tilde{\psi}}\left(A_{j} \omega\right)\right|^{2} d \omega<\varepsilon / 2
$$


By $\left(P_{1}\right),\left(P_{2}\right)$ and Lebesgue theorem, we have

$$
\lim _{k \rightarrow \infty} \sum_{j \geq J_{1}} \int_{A_{j}\left(\omega_{0}+H_{k}\right)}|\widehat{\tilde{\psi}}(\omega)|^{2} d \omega=0 .
$$

Thus for any $\varepsilon>0$, there exists $J_{1}$ such that $\lim _{k \rightarrow \infty}\left|R_{2,1}\left(f_{2}, g_{2}\right)\right| \leq \varepsilon$. Finally, we obtain

$$
\frac{1}{|\operatorname{det} B|} \sum_{(j, m) \in I\left(\alpha_{0}\right)} \widehat{\tilde{\psi}}\left(A_{j} \omega_{0}\right) \overline{\widehat{\psi}\left(A_{j}\left(\omega_{0}+\alpha_{0}\right)\right)}=0 \text {, for any } \alpha_{0} \in \wedge \backslash\{0\} \text {. }
$$

We complete the proof of Theorem 2 ,

\section{Remarks}

In the proof of Theorem 2, we have used the following result in some places.

Remark 3.1. If (2) and (3) holds, then there exist constants $C, q \in \mathbf{Z}_{+}$and $\beta \in(0,1)$ such that

(i) for any $p, j \in \mathbf{Z}_{+}, j \geq p q$ and $\xi \in \mathbf{R}^{n},\left|A_{j} \xi\right| \geq C\left(\frac{1}{\beta}\right)^{p}|\xi|$.

(ii) for any $p,-j \in \mathbf{Z}_{+}, j \leq-p q$ and $\xi \in \mathbf{R}^{n},\left|A_{j} \xi\right| \leq C \beta^{p}|\xi|$.

(iii) for any $j, k \in \mathbf{Z}, j<k$ and $\xi \in \mathbf{R}^{n}$, we have $\left|A_{k} \xi\right| \geq C\left(\frac{1}{\beta}\right)^{\frac{k-j}{q}}\left|A_{j} \xi\right|$.

Remark 3.2. Assume that $A$ is an expand matrix, $S=\Gamma \backslash \cup_{j=-\infty}^{-1}\left(A^{j} \Gamma\right)$, where $\Gamma$ is a bounded measurable subset in $\mathbf{R}^{n}, \sup _{x, y \in \Gamma}\|x-y\|<1$ and the origin is an interior point of $\Gamma$. Let $\widehat{\psi}=\chi_{S}$. By Theorem 4. $\psi$ is a tight frame function with respect to dilation sequence $\left\{A^{j}\right\}_{j \in \mathbf{Z}}$. If $D_{i}(i=1, \cdots, p)$ are nonsingular matrices which is commutative with $A$, and $A_{s p+i}=A^{s+1} D_{i}$ for any $s \in \mathbf{Z}, i=$ $1, \cdots, p$, then using Theorem 4 again, it is easy to show that $\psi$ is also a tight frame function with respect to dilation sequence $\left\{A_{j}\right\}_{j \in \mathbf{Z}}$.

\section{References}

1. Hernández, E., Weiss, G.: A first Course on Wavelets. CRC Press, Boca Raton (1996)

2. Frazier, M., Garrigos, G., Wang, K., Weiss, G.: A characterization of functions that generate wavelet and related expansion. J. Fourier Anal. Appl. 3 (1997) 883-906

3. Frazier, M., Jawerth, B., Weiss, G.: Littlewood-Paley theory and the study of function spaces. CBMS Regional Conference Series in Mathematics, 79, AMS, Providence, R1 (1991)

4. Yang, D., Zhou, X.: Wavelet frames with irregular matrix dilations and their stability. J. Math. Anal. Appl. 295 (2004) 97-106

5. Yang, D., Zhou, X.: Irregular wavelet frames on $L^{2}\left(\mathbf{R}^{n}\right)$. Science in China Ser. A Mathematics 2 (2005) 277-287

6. Yang, D., Zhou, X.: Frame wavelets with matrix dilations in $L^{2}\left(\mathbf{R}^{n}\right)$. Appl. Math. Letters 17 (2004) 631-639

7. Yang, X., Zhou, X.: An extension of Chui-Shi frame condition to nonuniform affine operations. Appl. Comput. Harmon. Anal. 16 (2004) 148-157 